only in a minority of patients. Prokinetics are widely used as first choice approach but treatment duration and stopping rules were not clearly established. Intrapyloric Botox injection showed subjective benefit in a proportion of patients, matching results of previous RCTs where placebo had similar benefit. Our study indicates the need for a more consistent and evidence-based management of gastroparesis. Dedicated outpatient clinics and internal protocols may help to achieve this task.

\section{PWE-135 CONSTIPATION IS THE MOST COMMON GASTROINTESTINAL SYMPTOM IN ACROMEGALY}

Nashiz Inayet*, Jamal Hayat, Gul Bano, Andrew Poullis. St George's Hospital and St George's, University of London, London, UK

\subsection{6/gutjnl-2018-BSGAbstracts.429}

Introduction Acromegaly is caused by a pituitary somatotroph adenoma resulting in excess secretion of growth hormone which leads to excess secretion of Insulin like growth factor 1 from the liver, causing abnormal soft tissue growth with a variety of symptoms and an increased risk of colorectal cancer. Somatostatin analogues, used in the management of acromegaly are also associated with a range of abdominal symptoms. We collected data from patients with a confirmed diagnosis of acromegaly to evaluate the frequency, type and burden of abdominal symptoms.

Methods Data was collected from patients with confirmed Acromegaly using SF36 RAND and Rome IV Diagnostic questionnaire and compared to a control group, to assess the burden of GI symptoms. Data analysis was carried out using Microsoft Excel and IBM SPSS v 25.

Results 49 acromegalics (23 male and 26 females; age range 23-64 years, mean 43) and 200 controls (92 male and 108 females; age range $18-84$, mean 42.4) were recruited. $94 \%$ (46 out of 49) of acromegalics reported abdominal symptoms and $79 \%$ (39 out of 49) had at least one FGID according to the Rome IV diagnostic criteria, compared to $36 \%$ of controls $(\mathrm{OR}>1, \mathrm{p}<0.0001)$. More acromegalic females reported suffering from abdominal symptoms (100\%) than males (87\%), however there was no significant difference observed in the frequency and intensity of symptoms. The most commonly reported symptom was constipation $(69 \%$ acromegalics vs $21 \%$ of controls OR $>1, \mathrm{p}<0.0001,95 \% \mathrm{CI} 4.4-15.8)$ followed by diarrhoea $(52 \%$ acromegalics vs $18 \%$ of controls OR $>1, \quad p<0.0001,95 \%$ CI $2.5-9.3) .30$ out of 49 (61\%) respondents met the criteria for Functional Constipation according to Rome IV. The prevalence of constipation increased with increasing age and was often associated with bloating. No statistically significant difference seen in the prevalence of upper GI, biliary and anorectal symptoms between the acromegaly patients and controls. Acromegalics scored lower on the mean scores of the eight parameters (physical functioning, role limitations due to physical health, role limitations due to emotional health, energy/fatigue, emotional wellbeing, social functioning, pain, general health (mean scores 60.04 vs 71.23 , 95\% CI -13.6829 to -8.6971 , OR $>1, p$ value $<0.001)$ measured by the RAND SF36 as compared to the control group.

Conclusions The presence of FGID affecting the lower gastrointestinal tract in acromegalics is substantially higher than the controls in our study. Factors such as delayed transit and increased bowel length may play a role. Functional Constipation is the most commonly reported problem. Poorer quality of life may in part be attributable to abdominal symptoms. Symptoms of the upper GI tract such as reflux, dyspepsia and vomiting do not appear to occur more frequently in this group as compared to controls.

\section{PWE-136 FUNCTIONAL GASTROINTESTINAL DISORDERS (FGID) IN EHLERS DANLOS TYPE III (HYPERMOBILE) AND MARFAN SYNDROME PATIENTS}

Nashiz Inayet*, Jamal Hayat, Maite Tome, Arvind Kaul, Ann Child, Andrew Poullis. St George's, University of London, London, UK

\subsection{6/gutjnl-2018-BSGAbstracts.430}

Introduction Ehlers Danlos syndrome is a group of inherited heterogenous multisystem disorders characterised by skin hyperextensibility, atrophic scarring, joint hypermobility and generalised tissue fragility. Hypermobile EDS (hEDS)is the most common type. Marfan syndrome(MS) is also a multisystem disorder caused by a mutation in FBN1 gene which shares some phenotypic features with Hypermobile EDS such as joint hypermobility. Recent studies have suggested an association of Functional Gastrointestinal Disorders with joint hypermobility.

Methods Data was collected from 27 MS patients (10 male and 17 females, age range 19-35 years mean 27) and 33 hEDS patients (3 male and 30 females, age range 19-32 years mean 23) with no organic gastrointestinal diagnosis, using SF36 RAND and Rome IV Diagnostic questionnaire and compared to control group (200 respondents, 92 male and 108 female; age range $18-84$, mean 42.4 ) to assess the burden of GI symptoms in these patients. Data analysis was carried out using Microsoft Excel and SPSS version 25 (IBM Corporation, America).

Results In both groups the majority $(78.3 \%)$ of respondents were female within the age range of 19-35 years. Both groups of patients showed a higher prevalence of abdominal symptoms as compared to the control group, however the hEDS group not only showed a higher prevalence but more frequent and severe symptoms meeting Rome IV criteria for diagnosis of FGIDs. 16 (49\%)of the EDS patients met the criteria for more than one FGID.

$\mathrm{p}$ values were significant $(\mathrm{p}<0.001)$ for functional heartburn, functional dyspepsia, functional dysphagia, IBS-D and functional bloating in hEDS patients when comparing the prevalence to controls.

The hEDS group also scored lower on quality of life scores (QOL) in comparison to either of the other groups with a mean score of 48.6 (95\%CI 25.3-33.4,p $<0.0001)$ as compared $54.2(95 \%$ CI $20.9-29.0, \mathrm{p}<0.0001)$ in the Marfan group and 78.6 in the control group.

Conclusions FGIDs are reported in both Marfan syndrome and Hypermobile Ehlers Danlos syndrome but appear to be more common and severe in hEDS. These patients score lower on quality of life scores as well despite hypermobility being a common feature of both conditions. Further research is needed in this area to see whether there are other factors that can explain this difference. 